\title{
Association between Grain and Legume Consumption and the Risk of Coronary Artery Obstruction among Jordanians Based on Angiography Results
}

\author{
Reema Tayyem ${ }^{1}$, Mohammed O. Ibrahim², Dana N. Abdelrahim³ ${ }^{3}$ Abdel-Ellah Al-Shudifat ${ }^{4}$, \\ Mohammed Azab ${ }^{4}$, Hadeel Ghazzawi ${ }^{3}$, and Hiba Bawadi ${ }^{1}$ \\ ${ }^{1}$ College of Health Sciences, QU-Health, Qatar University, Doha 2713, Qatar \\ ${ }^{2}$ Department of Nutrition and Food Technology, Faculty of Agriculture, Mu'tah University, Karak 61710, Jordan \\ ${ }^{3}$ Department of Nutrition and Food Technology, Faculty of Agriculture, The University of Jordan, Amman 11942, Jordan \\ ${ }^{4}$ Faculty of Medicine, The Hashemite University, Zarqa 13115, Jordan
}

\begin{abstract}
Accumulating evidence suggests that consumption of whole grains and legumes is associated with reduced risk of cardiovascular disease (CVD), whereas the risk is increased by consuming refined grains and cereals. This study aimed to investigate the association between grain and legume consumption and the risk of CVD. The study was conducted using a convenient sampling method with a total of 399 participants who underwent coronary angiography. Cases and controls were matched by age with a 1:1 ratio. Standardized and validated questionnaires were used to collect sociodemographic, health, lifestyle, and dietary data. Intake of more than 1 serving/d of white bread increased the risk of CVD significantly with an adjusted odds ratio (AOR) of 3.06 [95\% confidence interval (CI): 1.37 6.84], while consuming more than 1 serving/d of wholegrain bread reduced the risk significantly to approximately 53\% (AOR: $0.47,95 \%$ CI: 0.24 $\sim 0.93$ ). Similar trends between consuming white bread on daily basis and increased risk of CVD, and consuming wholegrain bread and reduced risk of CVD were also observed. In addition, consuming unsweetened cornflakes on a weekly basis had a protective effect against CVD (AOR: $0.15,95 \%$ CI: $0.03 \sim 0.96$ ). Intake of legumes reduced the risk of CVD, although only insignificantly for all the tested legumes. The present study calls for consideration of consuming wholegrain bread prevent CVD in the Jordanian population.
\end{abstract}

Keywords: cardiovascular disease, coronary angiography, grains, legumes

\section{INTRODUCTION}

The incidences of many non-communicable diseases (NCD), including diabetes mellitus (DM), hypertension, and cardiovascular diseases (CVDs), are increasing globally (GBD 2015 Mortality and Causes of Death Collaborators, 2016). CVDs are major contributors to the global burden of NCD, with an estimated 17.9 million people having died from CVDs in 2016, which represents 31\% of all global deaths [World Health Organization (WHO), 2017]. In 2018, the WHO attributed $37 \%$ of premature death in Jordan to CVDs (WHO, 2018). Age, sex, smoking, weight, the atherogenic diet, dyslipidaemia, physical inactivity, and diabetes are common risk factors for CVDs (Al-Shudifat et al., 2017).
Grains are the staple food for humankind worldwide and the primary constituent of many people's daily eating practice. Grains may provide $56 \%$ of daily energy intake and half of daily protein intake. The predominant types of grains consumed are wheat, corn, rice, oats, and rye. Wholegrains encompass endosperm, germ and bran; however refined grains do not contain the germ and bran, which are expelled during the processing procedure (Ye et al., 2012). Wholegrains and legumes are rich sources of fibre, B vitamins, and some trace minerals, including iron, magnesium, and zinc, which are condensed in the external bran layer (Oghbaei and Prakash, 2016).

In 2017, a meta-analysis demonstrated that wholegrain intake is related to a noteworthy decrease in the risks of coronary heart diseases (CHDs), CVDs, total cancer, and 
mortality from all causes (Aune et al., 2016). The metaanalysis showed that per $90 \mathrm{~g} / \mathrm{d}$ of wholegrains (three servings), the relative risk was 0.81 [95\% confidence interval (CI): 0.75 0.87, $\mathrm{n}=7$ studies] for $\mathrm{CHD}, 0.88$ (95\% CI: $0.75 \sim 1.03, \mathrm{n}=6)$ for stroke, and 0.78 (95\% CI: 0.73 0.85, $\mathrm{n}=10$ ) for CVD (Aune et al., 2016).

Although there is a converse relationship between high vs. low consumption of wholegrains and CVD, these amount have not been quantified (Flight and Clifton, 2006; Priebe and McMonagle, 2016). Subsequently, crosssectional studies cannot determine the amounts of wholegrains that should be consumed to protect against CVDs (Flight and Clifton, 2006; Priebe and McMonagle, 2016), and there may be a few reasons for conflicting dietary recommendations for wholegrain consumption. It is important to elucidate and give progressively more detailed and predictable dietary suggestions about the amounts and types of wholegrains that should be consumed to diminish the risk of chronic disease and premature mortality (Aune et al., 2016).

In addition, many studies have revealed that intake of legumes is inversely associated with CVD, myocardial infarction, cardiovascular mortality and non-cardiovascular mortality (Blekkenhorst et al., 2018; Papandreou et al., 2019).

Jordan is a developing country in the Middle East that has experienced changes in food consumption patterns among recent decades due to westernization (Alkurd et al., 2019). Despite these changes in food consumption patterns and practices, the most predominant source of energy is Jordan remains grains followed by legumes. Intake of grains and legumes in Jordan was reported as 174.1 and $5.96 \mathrm{~kg} /$ capita respectively, in a Household Expenditures and Income Survey conducted in 2010 [Department of Statistics (DOS), 2012]. The change in grains and legumes consumption from 1992 to 2010 was $-7.77 \%$ and $-30.25 \%$, respectively (DOS, 2012).

This case-control study was conducted to provide a comprehensive evaluation of the association between grains and legumes consumption and the risk of CVD.

\section{MATERIALS AND METHODS}

\section{Study participants and setting}

Participants were enrolled from the Catheterization Section of the Cardiology Department, Prince Hamzah Hospital (Amman, Jordan). A total of 399 patients who had undergone coronary angiography were recruited between January 2015 and December 2015. Cases and controls [ $n=$ 205 vs. $n=194$ (ratio 1:1), 160 females and 239 males] were matched by age (mean age of 52 years). All eligible patients were above 18 years of age, mentally and clinically stable, and underwent coronary angiography. The exclusion criteria included suffering from kidney, liver, or gastrointestinal diseases, and a previous diagnosis of CVD before angiography. Before collecting the data, instructions were given to participants about the study, and participants provided informed consent. This study was conducted according to the guidelines laid down in the Declaration of Helsinki and all procedures involving research study participants were approved by the IRB at Prince Hamza Hospital (IRB no. MH/32/2285). Written informed consent was obtained from all subjects/patients.

\section{Data collection}

Trained research assistants were responsible for recording information on participant's socio-demographic factors, previous health status (hypertension, DM, and dyslipidemia), smoking status, and family history of CVDs. Prior to the study, research assistants were selected, trained and given the standardized questionnaires (Tayyem et al., 2014). The data were collected before the participants underwent coronary angiography.

Coronary angiography: Coronary angiography was performed by a specialized cardiologist. The Seldinger technique was used to insert a catheter into the radial artery, whereby the tip was advanced to the aortic sinus cusp. To enable visualization of the arterial tree, a radio-contrast medium was passed through the coronary arteries and $\mathrm{X}$-rays were taken. To determine the area of narrowing, the degree of obstruction was estimated by comparing the arterial lumen to the adjacent normal artery and the value recorded in percentage. Participants with no stenosis $(0 \%)$ were enrolled as controls.

Dietary assessment: Intake of wholegrains and legumes was assessed for a year before the interview using validated Food Frequency Questionnaires (FFQs) (Tayyem et al., 2014), conducted by a trained dietitian. Most of the participants showed constant dietary patterns over the previous five years. Participants were asked to record their average frequently of consuming one standard serving of specific food items in nine categories below $(<1 /$ month, 2 3/month, 1 2/wk, 3 4/wk, 5 6/wk, 1/d, 2 3/d, $4 \sim 5 / \mathrm{d}$, or $6 / \mathrm{d}$ ) during the last year. The food groups were classified as grains (cooked rice, white bread, whole wheat bread, macaroni, bulgur, and unsweetened corn flakes) and legumes \{cooked beans, falafel [a deep-fried ball or a flat or doughnut-shaped patty made from ground chickpeas or fava beans (or both), herbs, spices, and onion], hummus, and legumes soup\}. To estimate portion size, food models, and standard measuring tools were used.

7-Day physical activity recall (PAR): A 7-day PAR validated questionnaire was used to calculate a participants' recall of time spent doing exercise over seven days (Sallis et al., 1985). This questionnaire helps to ascertain physical activity levels into 3 categories. 
Anthropometric measurements: Anthropometric (body weight, height, and waist circumference) measurements were taken by trained dietitians according to Lee and Nieman (2012). Body mass index (BMI) was calculated afterward by dividing weight in kilograms by the square of height in meters, and was categorized as normal body weight (18.5 $\sim 24.9)$, overweight $(25.0 \sim 29.9)$ and obese (>30.0) according to the National Institutes of Health.

\section{Statistical analysis}

SPSS version 20.0 software (IBM Corp., Armonk, NY, USA) was used for statistical analysis. Mean \pm standard error of the mean and percentages were used for descriptive statistics, with the significance level set at $P \leq 0.05$. The $95 \% \mathrm{CI}$ and the $P$-values for trends and adjusted odds ratios (AORs) were calculated using the multinomial logistic regression models and linear logistic regression models, respectively. Finally, based on reported CVDs risk factors, potential confounders (age, sex, BMI, smoking, physical activity, total energy intake, occupation, education level, marital status, and family history) were identified (Kerver et al., 2003).

\section{RESULTS}

A total of 399 participants who underwent elective coronary angiography were included in the study. The characteristics of study participants are summarized in a previously published article (Al-Shudifat et al., 2019). The majority of participants were male $(n=239,59.9 \%)$, and participants had a median age of 51 years. Mean fasting blood glucose levels in both male and female were higher in cases than controls. Additionally, blood triglyceride (TG) levels were higher in cases than controls, although this was only statistically significant for male. Significant differences in physical activity measured as metabolic equivalent (min/wk) were detected among the two groups; overall, cases were less active than controls, and reported more previous health problems than controls (Al-Shudifa et al., 2019). Intakes of white bread of over two servings/d significantly increased the risk of CVD with an AOR of 3.06 (95\%, CI: 1.37 6.84), whereas intakes of over two servings/d of wholegrain bread significantly reduced the risk to approximately $53 \%$ (AOR: 0.47 , $95 \%$ CI: $0.24 \sim 0.93$, Table 1 ). The same trends of increased risk of CVD with daily white bread consumption and reduced risk with daily wholegrain bread consumption is depicted in Table 2. However, no significant differences were detected for risk of CVD with the other tested grains and legumes except for the cornflakes. Consuming cornflakes on a weekly basis had a protective effect against CVD (OR: 0.15, 95\% CI: 0.03 0.96). However, consumption trends of all these foods were insignificant even within the white and wholegrain bread categories.

\section{DISCUSSION}

There is great interest in identifying the optimal diet for cardiovascular health. The global burden of disease studies considers diet as a crucial element behind the increasing number of people with diabetes, obesity, hypertension, and CVD. The present study aimed to examine the possible association between grains and legumes and the risk of CVD.

This results highlighted that higher consumption of white bread is associated with higher CVD risk. Moreover, it indicated that increased intake of wholegrain bread is associated with lower risk of developing CVD. These two findings are consistent with results reported in previous studies (Grundy et al., 2004; Hollænder et al., 2015). In a meta-analysis, Hollænder et al. (2015) showed that wholegrains lower the risk of developing CVD. Indeed, wholegrains lowered low density lipoprotein-cholesterol (LDL-C; weighted difference: $20.09 \mathrm{mmol} / \mathrm{L}, 95 \% \mathrm{CI}$ : $20.15 \sim 20.03 \mathrm{mmol} / \mathrm{L}, P_{\text {-Trend }}=0.01$ ) and total cholesterol (TC; weighted difference: $20.12 \mathrm{mmol} / \mathrm{L}, 95 \% \mathrm{CI}: 20.19$ $\left.\sim 20.05 \mathrm{mmol} / \mathrm{L}, P_{\text {-Trend }}=0.001\right)$ compared with controls (Hollænder et al., 2015). Furthermore, McKeown et al., (2002) conducted a cross-sectional study of 2,941 individuals aged 26 to 82 years to examine the association between diets rich in whole- and refined-grain and serum lipid profiles. The wholegrain intakes from the lowest to the highest quintile were inversely associated with TC levels $(5.21,5.14,5.25,5.18$, and $5.08 \mathrm{mmol} / \mathrm{L}$, respectively; $\left.P_{\text {-Trend }}=0.02\right)$, LDL-C levels $(3.17,3.14,3.22$, 3.17 , and $3.04 \mathrm{mmol} / \mathrm{L}$, respectively; $P_{\text {-Trend }}=0.006$ ), and TG levels $(1.70,1.60,1.61,1.66$, and $1.56 \mathrm{mmol} / \mathrm{L}$, respectively; $\left.P_{\text {-Trend }}=0.02\right)$. In addition, in a double-blind, randomized, and controlled crossover trial, 40 overweight or obese males and females aged $<50$ years without a history of CVD consumed wholegrain and refined grain diets for two 8-week periods. The authors reported significant reductions in systolic blood pressure, TC, LDL-C, body fat, and body weight following wholegrain consumption (Kirwan et al., 2016).

A meta-analysis of 10 prospective cohort studies (24 reports) was conducted by Mirrahimi et al. (2012) to investigate the potential associations between glycemic index (GI) or glycemic load (GL) with the incidence of CHD (Mirrahimi et al., 2012). The analysis indicated that the highest quantile had an increased risk of CHD vs. the lowest quantile for GI [relative risk (RR): 1.11, 95\% CI: 0.99 1.24] and GL (RR: 1.27, 95\% CI: 1.09 1.49). The higher dietary fiber content of wholegrains, especially of viscous soluble fiber ( $\beta$-glucans), contributes to these beneficial effects (Tighe et al., 2010). 
Table 1. Association between the consumed servings of cereals and legumes and CVD among the study participants

\begin{tabular}{|c|c|c|c|c|}
\hline \multirow[b]{2}{*}{ Food consumed } & \multicolumn{3}{|c|}{ Number of grain and legume servings consumed } & \multirow[b]{2}{*}{$P$-value } \\
\hline & $\begin{array}{l}<1 \text { serving } \\
\text { weekly }\end{array}$ & $1 \sim 6$ servings weekly & $>1$ serving daily & \\
\hline White bread & & & & 0.071 \\
\hline Case & 17 & 3 & 185 & \\
\hline Control & 25 & 7 & 162 & \\
\hline AOR $(95 \% \mathrm{CI})$ & 1 & $1.34(0.25 \sim 7.05)$ & $3.06(1.37 \sim 6.84)$ & \\
\hline Whole wheat bread & & & & 0.342 \\
\hline Case & 168 & 10 & 27 & \\
\hline Control & 153 & 8 & 33 & \\
\hline AOR $(95 \% \mathrm{CI})$ & 1 & $0.879(0.31 \sim 2.62)$ & $0.47(0.24 \sim 0.93)$ & \\
\hline Cooked rice & & & & 0.501 \\
\hline Case & 10 & 81 & 114 & \\
\hline Control & 4 & 95 & 95 & \\
\hline AOR (95\% CI) & 1 & $0.56(0.14 \sim 2.32)$ & $1.22(0.29 \sim 5.18)$ & \\
\hline Macaroni & & & & 0.354 \\
\hline Case & 157 & 48 & - & \\
\hline Control & 156 & 38 & - & \\
\hline AOR (95\% CI) & 1 & $1.33(0.75 \sim 2.35)$ & - & \\
\hline Bulgur & & & & 0.780 \\
\hline Case & 161 & 40 & 4 & \\
\hline Control & 150 & 40 & 4 & \\
\hline AOR (95\% CI) & 1 & $0.96(0.53 \sim 1.72)$ & $1.01(0.22 \sim 4.67)$ & \\
\hline Cooked beans & & & & 0.390 \\
\hline Case & 177 & 28 & - & \\
\hline Control & 173 & 21 & - & \\
\hline AOR (95\% CI) & 1 & $1.13(0.54 \sim 2.37)$ & - & \\
\hline Falafel & & & & 0.539 \\
\hline Case & 66 & 106 & 33 & \\
\hline Control & 60 & 97 & 37 & \\
\hline AOR (95\% CI) & 1 & $0.85(0.50 \sim 1.44)$ & $0.64(0.31 \sim 1.36)$ & \\
\hline Hummus & & & & 0.196 \\
\hline Case & 62 & 117 & 26 & \\
\hline Control & 50 & 112 & 32 & \\
\hline AOR (95\% CI) & 1 & $0.83(0.48 \sim 1.43)$ & $0.56(0.25 \sim 1.28)$ & \\
\hline Legumes coup (at winter) & & & & 0.171 \\
\hline Case & 32 & 94 & 77 & \\
\hline Control & 19 & 95 & 80 & \\
\hline AOR $(95 \% \mathrm{CI})$ & 1 & $0.64(0.30 \sim 1.40)$ & $0.65(0.28 \sim 1.52)$ & \\
\hline
\end{tabular}

AOR was adjusted for age, sex, total energy, physical activity, body mass index, education level, income level, marital status, family history of CVD, existence of health problem, and number of diseases.

CVD, cardiovascular disease; AOR, adjusted odds ratio; CI, confidence interval; -, not significant.

A meta-analysis of 22 prospective cohort studies showed that total dietary fiber consumption was inversely associated with risk of CVD (RR: 0.91, 95\% CI: 0.88 0.94) (Threapleton et al., 2013). Furthermore, in a cohort study conducted to assess the association between long-term dietary fiber intake and CHD, higher dietary fiber consumption was associated with lower risk of CHD. Indeed, each additional $10 \mathrm{~g}$ of recent dietary fiber intake was inversely associated with CHD [hazard ratio (HR): 0.83, 95\% CI: 0.70 0.98] (Streppel et al., 2008). The degree of cereal processing in addition to the fiber content may play a crucial role in the ability of wholegrains to aid prevention of CVD by modifying the impact of grain intake on the risk of CVD (Streppel et al., 2008). The refining process reduces the amount of dietary fiber due to removal of the bran and the germ, which leads to loss of their nutritional benefits, including reductions in blood pressure and blood cholesterol levels (Whelton et al., 2005). Moreover, this process enhances the bioavailability and accelerates the digestion of starch in the endosperm, which increases rapid glycemic responses (Ludwig, 2002). In addition, higher contents of minerals, phytochemicals, and fatty acids in wholegrains are likely to have major roles in explaining these inverse associations. The high contents of antioxidants in the wholegrains also clarifies the reversal effects on CVD (Călinoiu and Vodnar, 2018; 
Table 2. Association between the frequency of cereal and legume consumption and CVD among the study participants

\begin{tabular}{|c|c|c|c|c|c|}
\hline Food item & Rarely & Monthly & Weekly & Daily & $P$-value \\
\hline White bread & & & & & 0.123 \\
\hline Case & 16 & - & - & 186 & \\
\hline Control & 22 & - & - & 164 & \\
\hline AOR (95\% CI) & 1 & - & - & $0.89(1.16 \sim 4.87)$ & \\
\hline Whole wheat bread & & & & & 0.322 \\
\hline Case & 162 & & - & 29 & \\
\hline Control & 144 & & - & 34 & \\
\hline AOR $(95 \% \mathrm{CI})$ & 1 & & - & $1.02(0.41 \sim 2.50)$ & \\
\hline Cooked rice & & & & & 0.969 \\
\hline Case & 6 & - & 46 & 152 & \\
\hline Control & 3 & - & 53 & 138 & \\
\hline AOR $(95 \% \mathrm{CI})$ & 1 & - & $0.41(0.08 \sim 2.04)$ & $0.66(0.14 \sim 3.12)$ & \\
\hline Macaroni & & & & & 0.716 \\
\hline Case & 63 & 34 & 106 & - & \\
\hline Control & 68 & 20 & 106 & - & \\
\hline AOR $(95 \% \mathrm{CI})$ & 1 & $2.49(1.06 \sim 5.89)$ & $1.36(0.81 \sim 2.30)$ & - & \\
\hline Bulgur & & & & & 0.888 \\
\hline Case & 28 & 50 & 118 & 9 & \\
\hline Control & 33 & 36 & 117 & 8 & \\
\hline AOR $(95 \% \mathrm{CI})$ & 1 & $1.95(0.88 \sim 3.32)$ & $1.61(0.81 \sim 3.22)$ & $1.53(0.44 \sim 5.34)$ & \\
\hline Unsweetened cornflakes & & & & & 0.115 \\
\hline Case & 194 & 7 & 4 & - & \\
\hline Control & 179 & 3 & 12 & - & \\
\hline AOR $(95 \% \mathrm{CI})$ & 1 & $3.10(0.68 \sim 14.07)$ & $0.15(0.03 \sim 0.96)$ & - & \\
\hline Cooked beans & & & & & 0.468 \\
\hline Case & 64 & 108 & 31 & - & \\
\hline Control & 64 & 107 & 21 & - & \\
\hline AOR $(95 \% \mathrm{CI})$ & 1 & $0.97(0.58 \sim 1.64)$ & $1.44(0.65 \sim 3.18)$ & - & \\
\hline Falafel & & & & & 0.654 \\
\hline Case & 35 & 4 & 104 & 62 & \\
\hline Control & 26 & 3 & 113 & 52 & \\
\hline AOR $(95 \% \mathrm{CI})$ & 1 & $1.97(0.31 \sim 12.34)$ & $0.74(0.38 \sim 1.43)$ & $0.94(0.44 \sim 2.05)$ & \\
\hline Hummus & & & & & 0.801 \\
\hline Case & 30 & 4 & 117 & 54 & \\
\hline Control & 25 & 3 & 118 & 48 & \\
\hline AOR $(95 \% \mathrm{CI})$ & 1 & $1.47(0.22 \sim 9.67)$ & $0.86(0.43 \sim 1.69)$ & $0.95(0.43 \sim 2.10)$ & \\
\hline Legumes soup & & & & & 0.086 \\
\hline Case & 45 & 90 & 12 & 22 & \\
\hline Control & 28 & 94 & 13 & 27 & \\
\hline AOR $(95 \% \mathrm{CI})$ & 1 & $0.66(0.33 \sim 1.31)$ & $0.73(0.25 \sim 2.18)$ & $0.45(0.17 \sim 1.20)$ & \\
\hline
\end{tabular}

AOR was adjusted for age, sex, total energy, physical activity, body mass index, education level, income level, marital status, family history of CVD, existence of health problems, and number of disease.

CVD, cardiovascular disease; AOR, adjusted odds ratio; CI, confidence interval; -, not significant.

Francavilla and Joye, 2020). A previous study showed that phenolic compounds in wholegrains may inhibit LDL-C oxidation and platelet aggregation, two known risks for the incidence of CVD (Kris-Etherton et al., 2002). Also, wholegrains contain crucial minerals such as iron, zinc, copper, and selenium, which are considered indirect antioxidants that play key roles as cofactors of radical scavengers such as vitamin $\mathrm{E}$ and other antioxidant compounds that can stop chain reactions that lead to production of oxidative free radicals (Fardet, 2010). One of the proposed mechanisms for protective effects of wholegrains against hypertension is its ability to decrease gene expression of inflammatory markers while enhancing expression of anti-inflammatory markers (Borneo and León, 2012). Moreover, many other minerals present in wholegrains such as magnesium and potassium may help lower blood pressure (Lee et al., 2008).

No significant associations were detected between CVD and the other tested grains and legumes, except for the unsweetened cornflakes. Consuming cornflakes on a weekly basis had a protective effect against CVD (AOR: 0.20, 95\% CI: 0.04 0.97). From a nutritional point of view, legumes are considered an important source of antioxidants (such as vitamins and polyphenols), fiber, n-3 
fatty acids, bioactive compounds, and vegetable proteins (Bouchenak and Lamri-Senhadji, 2013). The results of our study showed that higher consumption of legumes such as falafel, hummus, and legumes soup is associated (but not significantly) with a lower risk of CVD development. These results are consistent with previous studies (Flock and Kris-Etherton, 2011). Indeed, Li et al. (2017) conducted a meta-analysis of six prospective cohort studies to explore the association between legume consumption and risk of CVD-associated mortality (Li et al., 2017). They did not find a statistically significant association between legume consumption and risk of CVD mortality. Through comparing the highest vs. lowest category, the pooled RR was 0.96 (95\% CI: 0.86 1.06, $I^{2}=38 \%, P_{\text {-Trend }}$ $=0.42$ ) for CVD mortality (Li et al., 2017).

However, Marventano et al. (2017) conducted a systematic review and meta-analysis of prospective cohort studies showing high legume intake was associated with decreased risk of 10\% for both CVD and CHD (RR: 0.90, 95\% CI: 0.84 0.97) (Marventano et al., 2017). The pooled analysis showed null results with no substantial decrease in the risk of stroke with increased intake of legumes (RR: 1.01, 95\% CI: 0.89 1.14). Marventano et al. (2017) stated that the impact of legumes on the risk of CVD and CHD may arise due to their intrinsic characteristics or because they are a substitute for unhealthy sources of protein. In contrast, Papandreou et al. (2019) documented that higher intake of legumes (chickpeas, lentils, and fresh peas) and dry beans was significantly associated with higher risk of CVD mortality, with HRs of 1.52 (95\% CI: $\left.1.02 \sim 2.89, P_{\text {-Trend }}=0.034\right), 2.23$ (95\% CI: $1.32 \sim 3.78, P_{\text {-Trend }}=0.002$ ), respectively (Papandreou et al., 2019).

One surprising result of our study was that frequent consumption of cornflakes had a protective trend against CVD development. In general, cornflakes are classified as a food with a high GI (Atkinson et al., 2008), which has been documented in previous randomized control trials and intervention studies to increase the risk of CVD in males and females with different outcomes (Yadav et al., 2016). Previous studies that support or contradict our finding are limited, although a recent study showed a different effect of cornflakes on lipid profiles. In the double-blind randomized study the effect of different types of flakes on serum lipid profiles of humans $(n=40)$ suffering from moderately raised serum cholesterol was investigated (Trinidad et al., 2004). The authors found that serum TC and LDL-C levels were significantly reduction following consumption of all types of flakes, except for cornflakes. Although cornflakes did not lower serum TC and LDL-C, they reduced serum TGs by $14.5 \%$, an effect that was comparable to other kinds of flakes. A possible explanation is that most consumed type of cornflakes is unsweetened with dried fruits cornflakes (as reported by our study participants). This type of cornflake can add nutritional value to the total fiber and antioxidants consumed by controls.

A main strength of this study is use of a validated Arabic FFQ, which was modified to reflect food consumption pattern in Arab countries, especially Jordan. Furthermore, use of food models and measuring tools to estimate portion sizes may have improved the accuracy of the collected data. A major limitation of this study was use of a dietary assessment tool that depends on memory recall. The one-year dietary recall period, which is what is usually used in FFQs, may not represent a sufficient amount of time to conclude an association between grain and legume consumption and CVD risk. The association between grain and legume consumption and CVD may have been developing for several years. However, we believe that the recall period of one year is likely representative of previous years. Another limitation was difficulty of matching females in case and control groups by age. However, we attenuated the effect possible differences in age by adjusting for age in all statistical analyses. Grouping as cases and controls was dependent on the results of the coronary angiography, which is not generally conducted for normal subjects. Even though sample sizes were relatively small, we calculated the required sample size to be approximately 385 subjects, which took one year to achieve.

In general, the findings of our study support the recommendation of increasing consumption of wholegrain bread as a protective factor against CVD. Daily wholegrain bread consumption was significantly correlated with reduced risk of developing CVD. However, daily consumption of refined bread was significantly associated with risk of CVD. The unexpected finding was the protective effect of unsweetened cornflakes against CVD.

\section{AUTHOR DISCLOSURE STATEMENT}

The authors declare no conflict of interest.

\section{REFERENCES}

Alkurd R, Takruri HR, Amr AM. Trends of energy and macronutrients intakes in Jordan as obtained by household expenditure and income surveys. J Agric Sci. 2019. 11:191-199.

Al-Shudifat AE, Azab M, Johannessen A, Agraib L, Tayyem RF. Is the intake of antioxidants associated with risk of coronary artery disease? A Jordanian case-control study. Top Clin Nutr. 2019. 34:259-268.

Al-Shudifat AE, Johannessen A, Azab M, Al-Shdaifat A, AbuMweis SS, Agraib LM, et al. Risk factors for coronary artery disease in patients undergoing elective coronary angiography in Jordan. BMC Cardiovasc Disord. 2017. 17:183. https://doi.org/10. 1186/s12872-017-0620-4 
Atkinson FS, Foster-Powell K, Brand-Miller JC. International tables of glycemic index and glycemic load values: 2008. Diabetes Care. 2008. 31:2281-2283.

Aune D, Keum N, Giovannucci E, Fadnes LT, Boffetta P, Greenwood DC, et al. Whole grain consumption and risk of cardiovascular disease, cancer, and all cause and cause specific mortality: systematic review and dose-response meta-analysis of prospective studies. BMJ. 2016. 353:i2716. https://doi.org/ 10.1136/bmj.i2716

Blekkenhorst LC, Sim M, Bondonno CP, Bondonno NP, Ward NC, Prince RL, et al. Cardiovascular health benefits of specific vegetable types: a narrative review. Nutrients. 2018. 10:595. https://doi.org/10.3390/nu10050595

Borneo R, León AE. Whole grain cereals: functional components and health benefits. Food Funct. 2012. 3:110-119.

Bouchenak M, Lamri-Senhadji M. Nutritional quality of legumes, and their role in cardiometabolic risk prevention: a review. J Med Food. 2013. 16:185-198.

Călinoiu LF, Vodnar DC. Whole grains and phenolic acids: a review on bioactivity, functionality, health benefits and bioavailability. Nutrients. 2018. 10:1615. https://doi.org/10.3390/ nu10111615

Department of Statistics (DOS). Household expenditures \& income survey 2010. DOS, Amman, Jordan. 2012.

Fardet A. New hypotheses for the health-protective mechanisms of whole-grain cereals: what is beyond fibre? Nutr Res Rev. 2010. 23:65-134.

Flight I, Clifton P. Cereal grains and legumes in the prevention of coronary heart disease and stroke: a review of the literature. Eur J Clin Nutr. 2006. 60:1145-1159.

Flock MR, Kris-Etherton PM. Dietary guidelines for Americans 2010: implications for cardiovascular disease. Curr Atheroscler Rep. 2011. 13:499-507.

Francavilla A, Joye IJ. Anthocyanins in whole grain cereals and their potential effect on health. Nutrients. 2020. 12:2922. https://doi.org/10.3390/nu12102922

GBD 2015 Mortality and Causes of Death Collaborators. Global, regional, and national life expectancy, all-cause mortality, and cause-specific mortality for 249 causes of death, 1980-2015: a systematic analysis for the Global Burden of Disease Study 2015. Lancet. 2016. 388:1459-1544.

Grundy SM, Cleeman JI, Merz CN, Brewer HB Jr, Clark LT, Hunninghake DB, et al.; National Heart, Lung, and Blood Institute; American College of Cardiology Foundation; American Heart Association. Implications of recent clinical trials for the National Cholesterol Education Program Adult Treatment Panel III guidelines. Circulation. 2004. 110:227-239.

Hollænder PL, Ross AB, Kristensen M. Whole-grain and blood lipid changes in apparently healthy adults: a systematic review and meta-analysis of randomized controlled studies. Am J Clin Nutr. 2015. 102:556-572.

Kerver JM, Yang EJ, Bianchi L, Song WO. Dietary patterns associated with risk factors for cardiovascular disease in healthy US adults. Am J Clin Nutr. 2003. 78:1103-1110.

Kirwan JP, Malin SK, Scelsi AR, Kullman EL, Navaneethan SD, Pagadala MR, et al. A whole-grain diet reduces cardiovascular risk factors in overweight and obese adults: a randomized controlled trial. J Nutr. 2016. 146:2244-2251.

Kris-Etherton PM, Hecker KD, Bonanome A, Coval SM, Binkoski $\mathrm{AE}$, Hilpert KF, et al. Bioactive compounds in foods: their role in the prevention of cardiovascular disease and cancer. Am J Med. 2002. 113:71S-88S.

Lee RD, Nieman DC. Nutritional assessment. 6th ed. McGrawHill, New York, NY, USA. 2012. p 167-169.

Lee YP, Puddey IB, Hodgson JM. Protein, fibre and blood pressure: potential benefit of legumes. Clin Exp Pharmacol Physiol. 2008. 35:473-476.
Li H, Li J, Shen Y, Wang J, Zhou D. Legume consumption and allcause and cardiovascular disease mortality. Biomed Res Int. 2017. 2017:8450618. https://doi.org/10.1155/2017/8450618

Ludwig DS. The glycemic index: physiological mechanisms relating to obesity, diabetes, and cardiovascular disease. JAMA. 2002. 287:2414-2423.

Marventano S, Izquierdo Pulido M, Sánchez-González C, Godos J, Speciani A, Galvano F, et al. Legume consumption and CVD risk: a systematic review and meta-analysis. Public Health Nutr. 2017. 20:245-254.

McKeown NM, Meigs JB, Liu S, Wilson PW, Jacques PF. Wholegrain intake is favorably associated with metabolic risk factors for type 2 diabetes and cardiovascular disease in the Framingham Offspring Study. Am J Clin Nutr. 2002. 76:390-398.

Mirrahimi A, de Souza RJ, Chiavaroli L, Sievenpiper JL, Beyene J, Hanley AJ, et al. Associations of glycemic index and load with coronary heart disease events: a systematic review and metaanalysis of prospective cohorts. J Am Heart Assoc. 2012. 1: e000752. https://doi.org/10.1161/JAHA.112.000752

Oghbaei M, Prakash J. Effect of primary processing of cereals and legumes on its nutritional quality: a comprehensive review. Cogent Food Agric. 2016. 2:1136015. https://doi.org/10.1080/ 23311932.2015.1136015

Papandreou C, Becerra-Tomás N, Bulló M, Martínez-González MÁ, Corella D, Estruch R, et al. Legume consumption and risk of all-cause, cardiovascular, and cancer mortality in the PREDIMED study. Clin Nutr. 2019. 38:348-356.

Priebe MG, McMonagle JR. Effects of ready-to-eat-cereals on key nutritional and health outcomes: a systematic review. PLoS One. 2016. 11:e0164931. https://doi.org/10.1371/journal. pone. 0164931

Sallis JF, Haskell WL, Wood PD, Fortmann SP, Rogers T, Blair SN, et al. Physical activity assessment methodology in the FiveCity Project. Am J Epidemiol. 1985. 121:91-106.

Streppel MT, Ocké MC, Boshuizen HC, Kok FJ, Kromhout D. Dietary fiber intake in relation to coronary heart disease and allcause mortality over $40 \mathrm{y}$ : the Zutphen Study. Am J Clin Nutr. 2008. 88:1119-1125.

Tayyem RF, Abu-Mweis SS, Bawadi HA, Agraib L, Bani-Hani K. Validation of a food frequency questionnaire to assess macronutrient and micronutrient intake among Jordanians. J Acad Nutr Diet. 2014. 114:1046-1052.

Threapleton DE, Greenwood DC, Evans CE, Cleghorn CL, Nykjaer C, Woodhead C, et al. Dietary fibre intake and risk of cardiovascular disease: systematic review and meta-analysis. BMJ. 2013. 347:f6879. https://doi.org/10.1136/bmj.f6879

Tighe P, Duthie G, Vaughan N, Brittenden J, Simpson WG, Duthie $S$, et al. Effect of increased consumption of whole-grain foods on blood pressure and other cardiovascular risk markers in healthy middle-aged persons: a randomized controlled trial. Am J Clin Nutr. 2010. 92:733-740.

Trinidad TP, Loyola AS, Mallillin AC, Valdez DH, Askali FC, Castillo JC, et al. The cholesterol-lowering effect of coconut flakes in humans with moderately raised serum cholesterol. J Med Food. 2004. 7:136-140.

Whelton SP, Hyre AD, Pedersen B, Yi Y, Whelton PK, He J. Effect of dietary fiber intake on blood pressure: a meta-analysis of randomized, controlled clinical trials. J Hypertens. 2005. 23:475481.

World Health Organization. Cardiovascular diseases (CVDs). 2021 [cited 2021 Sep 9]. Available from: https://www.who. int/news-room/fact-sheets/detail/cardiovascular-diseases(cvds)

World Health Organization. Noncommunicable diseases country profiles 2018. 2018 [cited 2020 Sep 9]. Available from: https://apps.who.int/iris/handle/10665/274512

Yadav D, Mishra M, Jadaun P, Choi E, Priyadharsini R, Kong ID. 
Glycemic index and glycemic load in cardiovascular disease risk. Progr Nutr. 2016. 18:95-101.

Ye EQ, Chacko SA, Chou EL, Kugizaki M, Liu S. Greater whole- grain intake is associated with lower risk of type 2 diabetes, cardiovascular disease, and weight gain. J Nutr. 2012. 142:13041313. 